\title{
Calidad de vida en niños y adolescentes sobrevivientes de leucemia linfoblástica aguda
}

\author{
VIOLETA CÁDIZ D. ${ }^{1 *}$, ALFONSO URZÚA M. ${ }^{2}$, MYRIAM CAMPBELL B. ${ }^{3}$ \\ 1. Psicóloga Clínica, Unidad de Oncología del Hospital Roberto del Río. Coordinadora subcomisión psicooncología PINDA. \\ 2. Psicólogo, Magister en Salud Pública, Doctor en Psicología Clínica y de la Salud, Escuela de Psicología, Universidad \\ Católica del Norte. \\ 3. Médico Hematoncólogo Pediatra, Unidad Oncología Hospital Roberto del Río. Coordinadora Protocolo Leucemia \\ Linfoblástica PINDA.
}

\begin{abstract}
Quality of life in children and adolescents surviving acute lymphoblastic leukemia

Objective: Describe the quality of life of patients cured of acute lymphoblastic leukemia (ALL) treated at the Oncology Unit at Roberto del Rio Children's Hospital. Method: With a transversal design, the Kidscreen-27 questionnaire was applied to a selected sample of 41 patients. Results were compared with a random sample of school children paired for age and sex. Results: Children cured from ALL had lower scores than the control group, although ANOVA showed differences were not significant. ALL children tended to self report as ill, significantly lower in physical well-being. Average followup time was 8 years. Conclusions: Quality of life of chilean children cured from ALL is similar to school children of similar age and gender. To preserve the quality of life, it is suggested that the following measures be taken: prevention of sequelae, detection and treatment of emotional disorders, and discharge at a reasonable time.
\end{abstract}

(Key words: Lymphoblastic leukemia, quality of life, cancer survivalr, Kidscreen-27).

Rev Chil Pediatr 2011; 82 (2): 113-121

\section{RESUMEN}

Objetivo: Describir la calidad de vida relacionada con la salud en niños curados de LLA tratados en la Unidad de Oncología del Hospital de Niños Roberto del Río. Método: Bajo un diseño transversal, se aplicó el cuestionario específico Kidscreen-27 a una muestra intencionada de 41 pacientes. Con fines ilustrativos se compararon, mediante un ANOVA, los resultados obtenidos con datos de dos sub-muestras pareadas en edad y sexo, escogidas mediante proceso de randomización en una muestra de niños chilenos escolarizados evaluados con el mismo instrumento. Resultados: Los niños curados de LLA tuvieron medias inferiores (no significativo) a la de los grupos con que se compararon. En la muestra estudiada se observó mayor proporción de niños 2010, tercera versión el 10 de enero de 2011, aceptado para publicación el 31 de enero de 2011.

Correspondencia a:

Dra. Violeta Cádiz D.

E-mail: violecadiz@gmail.com 
que se autoreportan como enfermos; en el ámbito del bienestar físico tienen una media significativamente inferior que los que se reportan sanos. El tiempo medio de control oncológico fue 8 años. Conclusiones: El auto-reporte de la calidad de vida de los niños curados de LLA es similar a una muestra de niños escolarizados chilenos. Para preservar la calidad de vida de los pacientes se sugiere que se realice prevención de secuelas, pesquisa y tratamiento de trastornos emocionales y alta definitiva en un plazo razonable.

(Palabras clave: Leucemia linfoblástica, calidad de vida, sobreviviente de cáncer, Kidscreen -27).

Rev Chil Pediatr 2011; 82 (2): 113-121

\section{Introducción}

La probabilidad de sobrevida de los niños con leucemia linfoblástica aguda (LLA) ha mejorado significativamente, obteniendo actualmente más de un $75 \%$ de curación ${ }^{1}$. En Chile esto se ha logrado debido a la mejora en el proceso diagnóstico, el desarrollo de protocolos de tratamientos de mayor efectividad, mejoría en el manejo de efectos adversos y complicaciones infecciosas asociadas, trabajo colaborativo multidisciplinario y la decisión de la autoridad sanitaria nacional de implementar acciones efectivas para la cura del cáncer como la creación del Programa Infantil Nacional de Drogas Antineoplásicas PINDA, implementado desde el año $1988^{2-4}$. Un niño se puede considerar curado cuando la probabilidad de morir por cáncer es similar a la que tiene un niño de la misma edad de morir por cualquier causa, aunque tenga secuelas secundarias a la enfermedad o al tratamiento recibido ${ }^{5}$. Cabe mencionar que el tiempo para "curar" depende del tumor, de sus factores biológicos y del tratamiento usado; en LLA sería entre 5 a 8 años desde el diagnóstico, manteniendo remisión completa continua. El tratamiento del cáncer involucra una combinación de quimioterapia, radioterapia y cirugía, con alto nivel de agresividad, que al ser aplicado en un niño, cuyo ser está en crecimiento, invade su desarrollo, lo altera y ocasiona impacto a nivel físico y psicológico, pudiendo dejar secuelas que se evidencian incluso tardíamente, afectando así su calidad de vida ${ }^{6-12}$. Al aumentar el número de niños curados de cáncer se genera la necesidad de evaluar el impacto que ha tenido la enfermedad en su calidad de vida, entendiendo por ésta el nivel de bienestar derivado de la evaluación que el menor realiza de diversos dominios de su vida, considerando el impacto que estos tienen en su estado de salud ${ }^{13}$. En esta evaluación se debe tomar en cuenta la habilidad de participar plenamente en las funciones y actividades relacionadas con aspectos físicos, sociales y psicosociales apropiadas para la edad ${ }^{14}$. La evaluación de la calidad de vida en niños sobrevivientes de cáncer infantil ha sido reportada en varios estudios ${ }^{15-17}$, sin embargo, el tipo de diseño utilizado no permite comparar los resultados obtenidos por los menores con una población similar que no haya sido afectada por la vivencia del cáncer. El objetivo de este trabajo es describir la calidad de vida percibida de los niños curados de LLA.

\section{Pacientes y Método}

La investigación corresponde a un tipo de estudio observacional de tipo transversal.

\section{Pacientes}

Se evaluaron niños y adolescentes, entre 8 y 18 años de edad, tratados por LLA, con protocolos PINDA, en la Unidad de Oncología del Hospital Roberto del Río, con más de 5 años en remisión completa continua desde el diagnóstico inicial. De acuerdo a la descripción de Haupt se consideran curados a los niños que cumplen estos requisitos ${ }^{5}$. Se incluyeron niños sin recaída y que no hubiesen recibido trasplante de médula. De 98 niños que cumplían con los requisitos de inclusión, fue posible contactar ya sea telefónicamente, por carta o coincidiendo con el control oncológico a 41 de ellos, todos los cuáles participaron voluntariamente. Los motivos por los que no se evaluaron los demás pacientes fueron ya sea que sus datos de contacto en la ficha no 
estaban actualizados, siendo muchos de ellos de regiones, o que el control se realizó fuera del plazo del estudio. Al ingreso al protocolo de LLA los padres firmaron un consentimiento que incluye todas las acciones necesarias para el tratamiento integral del cáncer, entre ellas el seguimiento biopsicosocial, el cual explicita que "El médico representante del equipo tratante nos han comunicado los detalles referentes al diagnóstico, tratamiento médico y/o quirúrgico, evolución de la enfermedad, seguimiento a largo plazo y posibilidad de curación así como de las posibles complicaciones que pueden aparecer tanto a consecuencia de la enfermedad como de los procedimientos diagnósticos y/o del tratamiento”.

\section{Instrumento}

Para evaluar la calidad de vida se utilizó el cuestionario Kidscreen-2 $27^{18}$, instrumento que ha sido utilizado en varios países europeos y en Chile ${ }^{19-20}$ y que presenta propiedades psicométricas adecuadas en niños y adolescentes chilenos $^{21}$. El Kidscreen-27 es un cuestionario genérico para niños con edades entre 8 y 18 años. Está conceptualmente basado en la definición de calidad de vida relacionada con la salud como un constructo multidimensional que evalúa cinco dimensiones:

Bienestar físico: inquiere sobre los niveles de actividad física, energía y estado físico (cinco ítems).

Bienestar psicológico: explora emociones positivas, satisfacción con la vida y sentimientos de equilibrio emocional (siete ítems).

Relación con los padres y autonomía: estudia la relación con los padres, atmósfera del hogar y sentimientos de tener la edad apropiada para independizarse y el grado de satisfacción con los recursos económicos (siete ítems).

Apoyo social y pares: evalúa la forma natural de relacionarse con otros niños/as y adolescentes (cuatro ítems).

Ambiente escolar: examina la percepción de su capacidad cognitiva, aprendizaje y concentración y sus sentimientos acerca de la escuela (4 ítems). Se usa tanto en población sana como en aquellos que padecen enfermedades crónicas.

\section{Procedimientos}

Una vez obtenidos los permisos correspondientes para la utilización del cuestionario por parte de sus creadores, previo revisión del lenguaje, se aplicó el cuestionario entre los meses de Diciembre de 2006 a Marzo de 2009, en coincidencia con la asistencia de los pacientes al control periódico en el Policlínico de Oncología. Todos los cuestionarios fueron administrados por la psicóloga de la Unidad; al momento de realizar el control anual del área psicosocial informó de la investigación al paciente y a su familiar acompañante, solicitando la participación y asegurando la confidencialidad de los datos personales. La administración tuvo un tiempo aproximado de 15 minutos permaneciendo el familiar fuera de la sala, generando así un clima de privacidad que favoreciera la veracidad de los menores al contestar la encuesta.

\section{Análisis de los datos}

Una vez aplicado el cuestionario, las respuestas fueron ingresadas a una base de datos construida en SPSS 17.0. Se calcularon los puntajes Rasch para cada dimensión transformándolos en valores, con una media en 50 y una desviación estándar de 10, siendo los puntajes más altos indicadores de mejor calidad de vida. Este es un modelo estadístico, diseñado por Rash, cuya fórmula matemática establece una relación entre la probabilidad de éxito y la diferencia entre la habilidad del individuo y la dificultad del ítem evaluado ${ }^{18}$. Las edades y tiempos se expresaron en medianas. Utilizamos el concepto genérico de "niño" para referirnos a niños/as y adolescentes. No se evaluó un grupo control, por lo que con fines ilustrativos para facilitar la comprensión de los resultados obtenidos, se compararon estos con una muestra pareada seleccionada de un estudio en 1678 niños escolarizados entre 8 y 18 años de edad, residentes en la ciudad de Antofagasta ${ }^{20}$. Para seleccionar éstos grupos de control, se dividió la muestra total en dos submuestras, una de aquellos niños que se percibían sanos y otra de aquellos que se percibían enfermos. $\mathrm{Al}$ interior de cada submuestra se realizó un proceso de randomización que consideró el $20 \%$ del total de los casos. Los grupos fueron 
completándose a medida que entre los casos randomizados iban encontrándose pares equivalentes al grupo de niños curados de LLA en cuanto a sexo y edad. Este procedimiento se realizó en ambas submuestras. Se agregó la pregunta " $¿$ Sufres de alguna enfermedad o defecto físico?", se denominó "estado de salud percibido" y fue utilizada como variable de análisis. Se realizaron los análisis descriptivos obteniéndose la media y desviación estándar (DE) de cada una de las dimensiones tanto para la muestra total como la estratificada entre aquellos que se percibían como enfermos y los que no. Se evaluaron las diferencias de medias entre estos dos últimos grupos a través de la prueba t de student. Posteriormente, se analizó la relación existente entre los puntajes obtenidos en la escala de calidad de vida, la edad y el tiempo en control oncológico a través del coeficiente de correlación de Pearson. Finalmente, a fin de evaluar la diferencia de las medias de los niños curados de LLA con pares de similar edad y sexo, se aplicó un análisis de varianza, ANOVA, para cada una de las dimensiones evaluadas.

\section{Resultados}

\section{Participantes}

De los 41 entrevistados, 23 fueron del sexo masculino. La media de edad fue de 13 años 6 meses (rango 8 años 9 m-18 años 7 m). Veintiocho pacientes (68\%) eran originarios de la Región Metropolitana y 13 (31\%) de la 3a - $4^{\mathrm{a}}$ región del país. Al diagnóstico, entre los años 1993 y 2000, tenían una edad media de 3 años 10 meses (rango 4 meses-13 años, $10 \mathrm{~m}$ ). La media de tiempo transcurrido entre el diagnóstico de LLA y la administración del cuestionario, se denominó "tiempo en control oncológico” y fue de 8 años 1 mes (rango 5 años-13 años 11 meses). Los grupos a comparar fueron pareados en cuanto a edad y sexo, de acuerdo a los procedimientos explicados, con excepción del total de mujeres de 18 años percibida como enferma en el grupo de comparación, ya que no fue posible asignar un par (tabla 1).

\section{Autoreporte de la calidad de vida}

La tabla 2 muestra los puntajes obtenidos por los menores evaluados en las distintas

Tabla 1. Distribución por sexo y edad en niños curados de LLA y en los grupos de comparación

\begin{tabular}{|c|c|c|c|c|c|c|}
\hline \multirow[t]{2}{*}{$\begin{array}{l}\text { Edad } \\
\text { (años) }\end{array}$} & \multicolumn{2}{|c|}{$\begin{array}{c}\text { Menores Curados } \\
\text { LLA }\end{array}$} & \multicolumn{2}{|c|}{$\begin{array}{l}\text { Grupo comparación } \\
\text { percepción estar sanos }\end{array}$} & \multicolumn{2}{|c|}{$\begin{array}{l}\text { Grupo comparación } \\
\text { percepción estar enfermos }\end{array}$} \\
\hline & Hombre & Mujer & Hombre & Mujer & Hombre & Mujer \\
\hline 8 & 3 & 1 & 3 & 1 & 3 & 1 \\
\hline 9 & 2 & 2 & 2 & 2 & 2 & 2 \\
\hline 10 & 2 & 1 & 2 & 1 & 2 & 1 \\
\hline 11 & 4 & 2 & 4 & 2 & 4 & 2 \\
\hline 12 & 2 & 1 & 2 & 1 & 2 & 1 \\
\hline 13 & 1 & 0 & 1 & 0 & 1 & 0 \\
\hline 14 & 2 & 1 & 2 & 1 & 2 & 1 \\
\hline 15 & 2 & 1 & 2 & 1 & 2 & 1 \\
\hline 16 & 3 & 3 & 3 & 3 & 3 & 3 \\
\hline 17 & 2 & 4 & 2 & 4 & 2 & 4 \\
\hline 18 & 0 & 2 & 0 & 2 & 0 & 1 \\
\hline Total & 23 & 18 & 23 & 18 & 23 & 17 \\
\hline
\end{tabular}

LLA = Leucemia linfoblástica aguda. 
Tabla 2. Medias y DE de cada dimensión para el total de niños curados de LLA y estratificados por percepción de salud/ enfermedad

\begin{tabular}{|c|c|c|c|}
\hline Dimensión & $\begin{array}{c}\text { Menores curados } \\
\text { LLA } \\
\text { Media } \pm \text { DE } \\
n=41\end{array}$ & $\begin{array}{c}\text { Percepción de estar } \\
\text { enfermo } \\
\text { Media } \pm \text { DE } \\
n=19\end{array}$ & $\begin{array}{c}\text { Percepción de estar } \\
\text { sano } \\
\text { Media } \pm \text { DE } \\
n=22\end{array}$ \\
\hline Bienestar físico & $44,71 \pm 7,84$ & $42,05 \pm 8.17^{*}$ & $47,01 \pm 6,91$ \\
\hline Bienestar psicológico ${ }^{1}$ & $40,04 \pm 3,62$ & $39,43 \pm 4,19$ & $40,58 \pm 3,05$ \\
\hline Relación padres y autonomía' & $45,40 \pm 10,60$ & $44.14 \pm 10,47$ & $46,49 \pm 10,85$ \\
\hline Apoyo social y pares ${ }^{1}$ & $49,60 \pm 8,75$ & $47,27 \pm 8,58$ & $51,62 \pm 8,57$ \\
\hline Ambiente escolar ${ }^{1}$ & $49,54 \pm 12,96$ & $48,01 \pm 14,53$ & $50,87 \pm 11.62$ \\
\hline
\end{tabular}

LLA = Leucemia linfoblástica aguda; $\mathrm{DE}=$ Desviación estándar; $\mathrm{n}=$ número de participantes evaluados; *Media significativamente menor que la del grupo que se percibe como sano, con una probabilidad de error del $5 \%$. ${ }^{1}$ Diferencia entre medias obtenidas por cada grupo en esta dimensión no es significativa.

Tabla 3. Auto-reporte de la calidad de vida en niños curados de LLA, distribuidos según sexo

\begin{tabular}{|lcc|}
\hline Dimensión Kidscreen-27 & \multicolumn{1}{c|}{$\begin{array}{c}\text { Nomos curados de LLA } \\
\text { media } \pm \text { DE2 } \\
\mathbf{n}=\mathbf{2 3}\end{array}$} & $\begin{array}{c}\text { Mujeres } \\
\text { media } \pm \text { DE } \\
\mathbf{n}=\mathbf{1 8}\end{array}$ \\
\hline Bienestar físico' $^{1}$ & $46,50 \pm 7,64$ & $42,43 \pm 7,69$ \\
\hline Bienestar psicológico' $^{1}$ & $39,46 \pm 3,00$ & $40,80 \pm 4,26$ \\
\hline Relación con padres y autonomía $^{1}$ & $47,54 \pm 9,62$ & $42,66 \pm 11,44$ \\
\hline Apoyo social y pares $^{1}$ & $49,65 \pm 8,90$ & $49,54 \pm 8,81$ \\
\hline Ambiente escolar' $^{1}$ & $49,58 \pm 14,16$ & $49,51 \pm 11,65$ \\
\hline
\end{tabular}

LLA = Leucemia Linfoblástica Aguda; DE=Desviación Estándar; $n=$ número de participantes evaluados. 'Diferencia entre medias obtenidas por cada sexo en esta dimensión no es significativa.

dimensiones que incorpora el instrumento. A nivel de la muestra total, el dominio peor evaluado fue el del bienestar psicológico y los mejor evaluados los de apoyo social y pares y ambiente escolar. Esta evaluación se repite al separar la muestra entre aquellos que se percibían sanos y aquellos que se percibían enfermos. Tal como se observa, los puntajes de aquellos menores que se autoreportaron como enfermos, son menores que aquellos que se evaluaron como sanos en todas las dimensiones, siendo estadísticamente significativa sólo en la dimensión del bienestar físico $(\mathrm{p}<, 05)$.

La tabla 3 muestra las medias obtenidas en las distintas dimensiones al estratificar el análisis por sexo. Tal como se puede observar, las medias en los hombres son superiores a las de las mujeres en todas las dimensiones con excepción del bienestar psicológico, pero ninguna de estas diferencias es significativa.

$\mathrm{Al}$ evaluar la relación existente entre la edad de los participantes con el puntaje obtenido en las diversas dimensiones, se encuentra que esta se correlaciona de manera inversa solamente con el dominio físico $(r=-, 471$; $(\mathrm{p}<, 05)$, en donde a mayor edad, es menor la calidad de vida percibida en esta dimensión (figura 1). Al analizar la relación existente entre el puntaje obtenido en las distintas dimensiones y el tiempo en control oncológico, éste correlaciona de manera inversa con el bienestar físico $(r=-, 440 ; p<, 05)$, el bienestar psi- 
CÁDIZ V. y cols.

Tabla 4. Medias y desviaciones estándar de los niños curados de LLA comparados con los niños escolarizados que se autoevalúan como sanos y enfermos

\begin{tabular}{|lccc|}
\hline Dimensión & $\mathbf{n}=\mathbf{4 1}$ & $\begin{array}{c}\text { Menores curados LLA } \\
\text { percepción buena salud } \\
\mathbf{n = 4}\end{array}$ & $\begin{array}{c}\text { Grupo de comparación } \\
\text { percepción enfermos } \\
\mathbf{n}=\mathbf{4 0}\end{array}$ \\
\hline Bienestar físico' $^{1}$ & $44,71 \pm 7,84$ & $47,21 \pm 9,16$ & $45,06 \pm 7,25$ \\
\hline Bienestar psicológico' & $40,04 \pm 3,62$ & $40,91 \pm 3,45$ & $41,17 \pm 3,26$ \\
\hline Relación padres y autonomía' & $45,40 \pm 10,60$ & $48,86 \pm 8,82$ & $46,14 \pm 7,53$ \\
\hline Apoyo social y pares' & $49,60 \pm 8,75$ & $51,62 \pm 9,30$ & $53,09 \pm 9,01$ \\
\hline Ambiente escolar' & $49,54 \pm 12,96$ & $51,23 \pm 9,85$ & $49,93 \pm 8,96$ \\
\hline
\end{tabular}

LLA = Leucemia linfoblástica aguda; $\mathrm{DE}=$ Desviación estándar; $\mathrm{n}=$ número de participantes evaluados; ${ }^{1}$ Diferencia entre medias obtenidas por cada sexo en esta dimensión no es significativa.

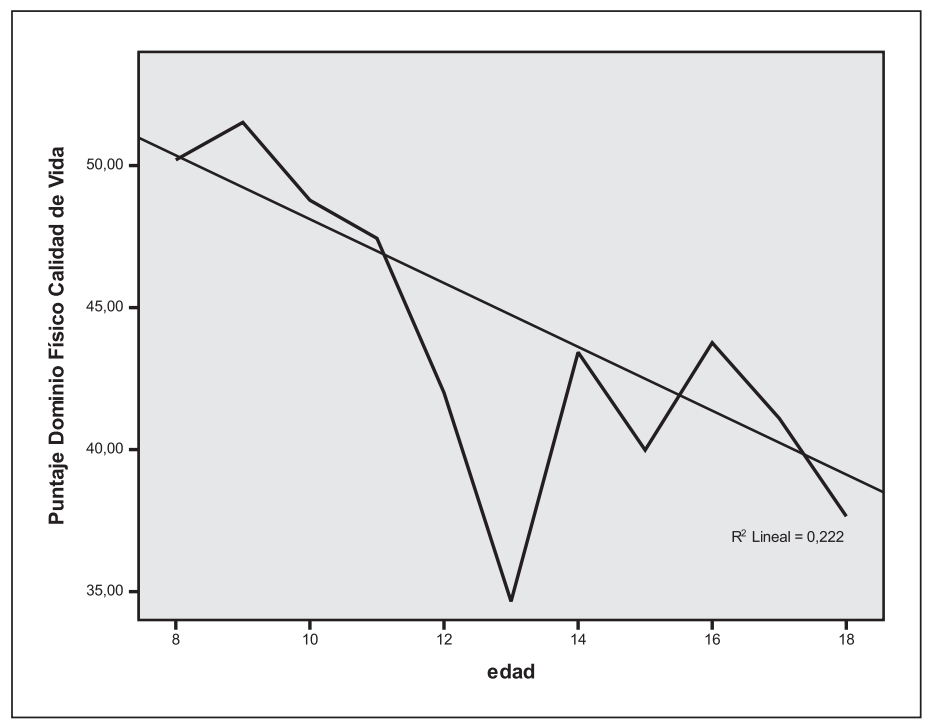

Figura 1. Correlación edad y dominio físico.

cológico $(\mathrm{r}=-, 386 ; \mathrm{p}<, 05)$ y el apoyo social y pares $(r=-, 316 ; \mathrm{p}<, 05)$, de manera que a mayor tiempo en control oncológico peor es la calidad de vida percibida en estas dimensiones (figura 2). Los resultados de las medias en todas las dimensiones, reportados por los niños curados de LLA son inferiores al compararlas con los subgrupos de los niños escolarizados chilenos que se perciben sanos y enfermos, aunque estas diferencias no son significativas. Sin embargo, la proporción de niños que se autoevalúan como enfermos es significativamente mayor en los curados de LLA (19/41, $46,3 \%)$ que en el estudio de niños escolariza$\operatorname{dos}(264 / 1678,15,7 \% \mathrm{p}<, 01)$.

\section{Discusión}

El cáncer es una patología que provoca un gran impacto en el paciente y en su familia, especialmente durante la etapa de tratamiento, sin embargo, algunos de sus efectos pueden hacerse más evidentes en la etapa de curación. Estas secuelas o efectos tardíos pueden ser provocados por la enfermedad o la terapia recibida y pueden afectar tanto el aspecto físico como psicológico ${ }^{22-25}$. Las secuelas psicosociales se refieren a alteraciones que afectan los sistemas familiares y/o individuales de cada paciente como el desarrollo de la personalidad, aspectos cognitivos, relación con sus 


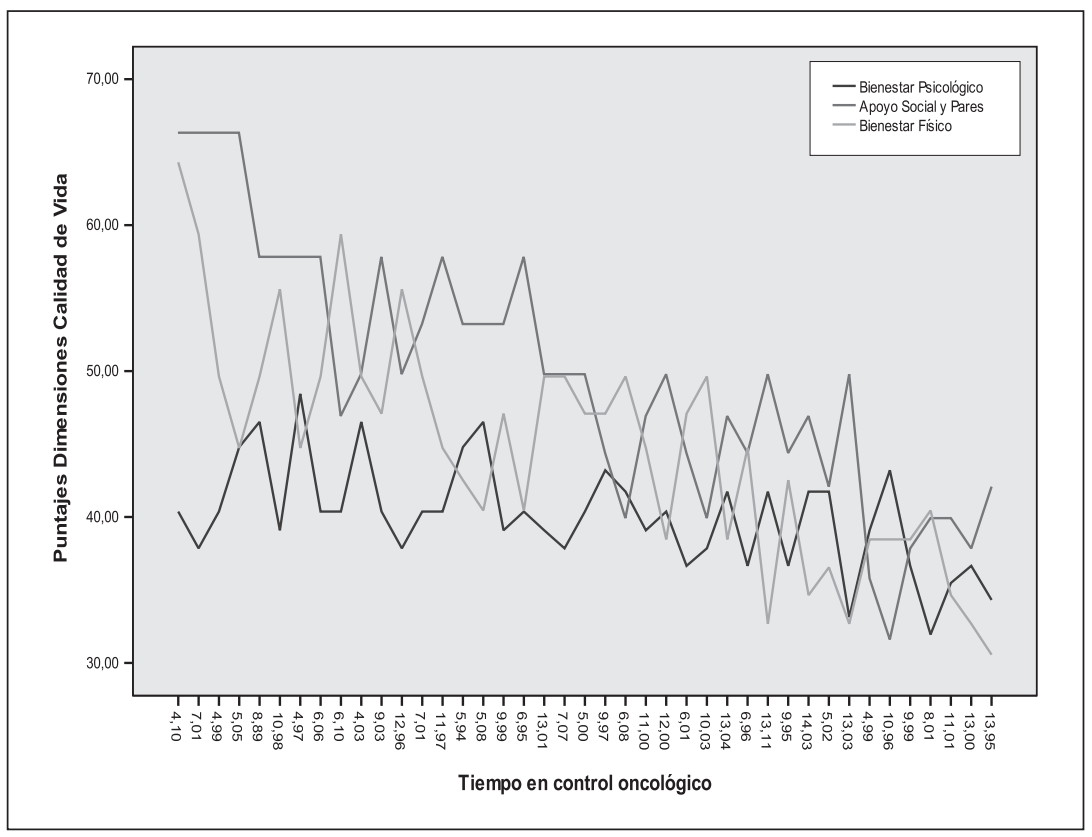

Figura 2. Correlación de puntajes dimensiones calidad de vida y tiempo en control oncológico. pares, vida escolar, laboral, percepción del futuro y metas de vida entre otros. Estas secuelas pueden ser físicas, como amputación de un miembro, o también surgir a partir de un trastorno emocional en sí asociado a las vivencias traumáticas vividas por el niño y la familia durante el tratamiento. El síndrome de Damocles, o temor a la recaída, es una de las secuelas psicosociales más frecuentes generando en los padres una actitud de sobreprotección y control excesivo hacia el paciente, afectando el desarrollo del crecimiento personal y autonomía; los pacientes también pueden ver afectada su seguridad vital mostrando sentimientos de vulnerabilidad, inseguridad en sí mismo, temor a perder la salud, a la muerte, o sentimientos de inferioridad al compararse con sus pares; los sistemas familiares frecuentemente sufren alteraciones surgiendo disfunciones en los diferentes sub-sistemas ${ }^{10}$. En este estudio el auto-reporte de la calidad de vida de los niños curados de LLA es similar a la de los niños escolarizados chilenos, aunque algunos de ellos tengan efectos tardíos. Los hallazgos de este estudio son similares a otros publicados ${ }^{26}$, sin embargo, muchos incluyen sobrevivientes de cualquier cáncer en cambio ésta es una mues- tra seleccionada y homogénea de pacientes, con una patología oncológica con alta posibilidad de curación, que no incluyó pacientes con recaída, trasplante o con tumores de peor pronóstico como por ejemplo los del sistema nervioso central en los que probablemente los resultados serían diferentes. Respecto a la auto-percepción de sentirse enfermo o con algún defecto físico, esto se asocia a una peor calidad de vida, correlacionándose inversamente con el bienestar físico, relación que también se observa en la población sana. Esto es comprensible si se piensa que tanto en el grupo de niños curados de LLA como en la población normal, existe un número que presenta problemas de salud de diversa índole. Todos los niños curados de LLA, que refirieron tener problemas de salud, reconocieron que tipo de patología tenían siendo la gran mayoría de ellas clínicamente comprobable y al menos algunas de ellas podrían estar relacionadas con el tratamiento la leucemia (hipotiroidismo, obesidad, cicatriz de catéter) y podría explicar la proporción mayor de autoreporte de "enfermos" en relación a la población escolarizada sana. Del mismo modo la experiencia de haber tenido una enfermedad grave, con padres aprensivos 
y en control médico sistemático puede favorecer la percepción de enfermedad. Respecto a la variable edad, se observa que a mayor edad, hay una menor calidad de vida percibida en relación al bienestar físico, relación similar a lo que ocurre en la población normal, y que puede explicarse por la cercanía de la adolescencia, etapa caracterizada por ser crítica en cuanto a transformaciones, tanto corporales como psicológicas, asociados a una actitud a menudo cuestionadora de la realidad y de la autoimagen. Esta misma relación existe en el grupo con mayor tiempo en control oncológico que refiere menor calidad de vida percibida en su bienestar físico, psicológico y relación con sus pares. Se sugiere que para preservar la calidad de vida de los pacientes es recomendable que el equipo esté preocupado de realizar prevención de secuelas, pesquisa y tratamiento de los trastornos emocionales en la etapa del tratamiento, notificar el fin del tratamiento y el alta definitiva de la Unidad de Oncología en un plazo razonable.

Si bien en todos los pacientes se han realizado los controles médicos de seguimiento a largo plazo, el haber evaluado calidad de vida sólo en un grupo de los sobrevivientes, por las razones ya explicadas, podría limitar la extensión de los resultados a sólo la población estudiada. Este punto se convierte en un elemento a considerar en la planificación y desarrollo de futuras investigaciones que permitan comprender como evoluciona la calidad de vida post alta.

Pese a esto, esta investigación posibilita disponer de evidencia que permite ir avanzando en la comprensión de la LLA en población infantil y adolescente, con miras a optimizar los protocolos de intervención basados en la evidencia.

\section{Agradecimientos}

A las psiquiatras, Dras. Viviana Herskovic y Catalina Poblete, por haber iniciado este trabajo contactándose con el Grupo Europeo Kidscreen y haber realizado los cambios necesarios en el lenguaje del texto chileno. Al Dr. Luis Rajmil, del IMIM en Barcelona, quién nos asesoró acerca de la utilización de este instrumento y a los hematoncólogos pediatras de PINDA, Dres. Lautaro Vargas y Ana Becker, coordinadora del Protocolo de Seguimiento Biopsicosocial PINDA.

\section{Referencias}

1.- Gatta G, Capocaccia R, Stiller C, et al: Childhood cancer survival trends in Europe: EUROCARE Working Group study. J Clin Oncol 2005; 23: 3742-51.

2.- Becker A \& PINDA Chile: Pronóstico de vida y secuelas del tratamiento del cáncer en los niños. Rev Chil Pediatr 2003; 74 (5): 520-3.

3.- Vargas L: Cáncer en pediatría: Aspectos generales. Rev Chil Pediatr 2000; 71 (4): 283-95.

4.- Campbell M: Desarrollo de la Oncología Pediátrica en Chile. Rev Ped Elec 2005; 2 (2): 1-4.

5.- Haupt R, Spinetta J, Ban I, et al: Long term survivors of childhood cancer: cure and care. European Journal of cancer 2007; 43: 1778-80.

6.- Soliman H, Agresta S: Current Issues in adolescent and young adult cancer survivorship. Cancer Control 2008; 15 (1): 55-62.

7.- Vance $Y$, Morse R: Issues in measuring Quality of life in childhood cancer: measures, proxies, and parental mental health. J Child Psychol Psychiat 2001; 42 (5): 661-7.

8.- $\quad$ Landolt M, Vollrath $M$, Niggli F, Gnehm H, Sennhauser $F$ : Health related quality of life in children with newly diagnosed cancer: a one year follow-up study. Health and Quality of Life Outcomes 2006; 4 (1): 63-70.

9.- Malhotra S, Singh G: Psychological consequences of chronic physical illnesses in children and adolescents. Indian Journal of pediatrics 2002; 69: 145-8.

10.- Mulhern R, Phipps S, White H: Neuropsychological outcomes. En Wallace H and Green D (Eds). Late effects of childhood cancer. Arnold. London 2004; 18-36.

11.- White E: Quality of life and body image. En Wallace $\mathrm{H}$ and Green D (Eds). Late effects of childhood cancer. Arnold. London 2004; 335-49.

12.- Jóhannsdóttir I, Hjermstad M, Moum T, et al: Social outcomes in young adult survivors of low incidence childhood cancers. J Cancer Surviv 2010; DOI 10.1007/ S11764-009-0112-3.

13.- Langeveld N, Stam H, Grootenhuis M, Last B: Quality of life in young adult survivors of childhood cancer. Support Care Cancer 2002; 10: 579-600.

14.- Urzúa A: Calidad de Vida relacionada con la Salud: elementos conceptuales. Rev Med Chile 2010; 138: 341-8. 
15.- Pogorzala M, Styczynski J, Kurylak A, Debski R, Wojtkiewicz $M$, Wysocki $M$ : Health related quality of life among paediatric survivors of primary brain tumours and acute leukaemia. Qual Lif Res 2010; 19: 191-8.

16.- Vaudre G, Trocmé N, Landman J, et al: Vécu des adolescents guéris d'une leucémie aiguë Lymphoblastique. Archives de Pédiatrie 2005; 12: 1591-9.

17.- Zebrack B, Chesler M: A Psychometric analysis of the quality of life cancer survivors (QOL-CS) in survivor of childhood cancer. Quality of life research 2001; 10 : 319-29.

18.- Ravens-Sieberer U, Gossh AA, Rajmil L (coordinador España) \& The European KIDSCREEN Group: (2007). Quality of life questionnaires for children and adolescents. Handbook. Pabst Science Publishers. Lengerich, Alemania

19.- Ravens-Sieberer U, Auquier P, Erhart M, The European KIDSCREEN Group: The KIDSCREEN-27 quality of life measure for children and adolescents: psychometric results from a cross-cultural survey in 13 European countries. Quality of Life Research 2007; 16 (8): 134756.

20.- Urzúa A, Cortés E, Prieto L, Vega S, Tapia K: Autoreporte de la Calidad de Vida en Niños y adolescentes Escolarizados. Rev Chil Pediatr 2009; 80: 238-44.
21.- Urzúa A, Cortés E, Vega S, Prieto L, Tapia K: Propiedades Psicométricas del Cuestionario de Auto Reporte de la Calidad de Vida Kidscreen-27 en adolescentes chilenos. Terapia Psicológica 2009; 27: 83-92.

22.- Becker $A$, Vargas $L$ : ¿Por qué y para qué el seguimiento a largo plazo? En: Becker A, Vargas L. ed. Dejé atrás el cáncer, una guía para el futuro. Fundación Niño y Cáncer, PINDA-Santiago Chile 2004: 27-32.

23.- Rona E, Cádiz V: ¿Qué reacciones emocionales se observan en niños y adolescentes curados de cáncer? En: Becker A, Vargas L. ed. Dejé atrás el cáncer, una guía para el futuro. Fundación Niño y Cáncer, PINDASantiago Chile 2004: 39-46.

24.- Cádiz V, Rona E: Problemas psicológicos después de finalizado el tratamiento en el niño y su familia. En: Becker A, Vargas L. ed. Dejé atrás el cáncer, una guía para el futuro. Fundación Niño y Cáncer, PINDASantiago Chile 2004: 47- 65.

25.- Keene N, Hobbie W, Ruccione K: O’Reilly. Survivorship En Childhood Cancer Survivors, a practical guide to your future. Editorial O’Reilly 2000.

26.- Harila M, Salo J, Lanning M, Vilkkumaa I, HarilaSaari A: Health related quality of life among long term survivors of childhood acute lymphoblastic leukemia. Pediatr. Blood Cáncer 2010; 55: 331-6. 